\title{
Rodent Model of Disease
}

National Cancer Institute

\section{Source}

National Cancer Institute. Rodent Model of Disease. NCI Thesaurus. Code C19021.

A rodent disease whose pathologic mechanisms are sufficiently similar to those of a human disease to serve as a model. 